\title{
Erratum to: High-throughput fast full-color digital pathology based on Fourier ptychographic microscopy via color transfer [Sci. China-Phys. Mech. Astron. 64(11), 114211 (2021)]
}

\author{
Yuting Gao ${ }^{1,2}$, Jiurun Chen ${ }^{1,2}$, Aiye Wang ${ }^{1,2}$, An Pan $^{1 *}$, Caiwen Ma ${ }^{1 *}$, and Baoli Yao ${ }^{1,2}$ \\ ${ }^{1} X i$ 'an Institute of Optics and Precision Mechanics, Chinese Academy of Sciences, Xi'an 710119, China; \\ ${ }^{2}$ University of Chinese Academy of Sciences, Beijing 100049, China
}

Received August 2, 2021; accepted August 9, 2021; published online October 26, 2021

Erratum to: Sci. China-Phys. Mech. Astron. 64(11), 114211 (2021), https://doi.org/10.1007/s11433-021-1730-x

Citation: $\quad$ Y. Gao, J. Chen, A. Wang, A. Pan, C. Ma, and B. Yao, Erratum to: High-throughput fast full-color digital pathology based on Fourier ptychographic microscopy via color transfer [Sci. China-Phys. Mech. Astron. 64(11), 114211 (2021)], Sci. China-Phys. Mech. Astron. 64, 124251 (2021), https://doi. org/10.1007/s11433-021-1762-8

In this paper [1], Figure 5(a) is mistakenly used with the figure of ground truth, which is taken and stitched by $10 \times 1$ $0.3 \mathrm{NA}$ objective and the operations have been detailed in the main text. The correction is as follows (Figure 1). This error does not affect the conclusion of this paper. The authors have also checked other figures such as Figure 6, and they are used correctly.

1 Y. Gao, J. Chen, A. Wang, A. Pan, C. Ma, and B. Yao, Sci. ChinaPhys. Mech. Astron. 64, 114211 (2021).

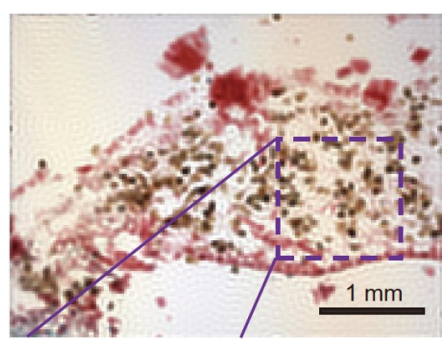

Figure 1 (Color online) LR donor image with the entire FOV of a $4 \times / 0.1 \mathrm{NA}$ objective.

The online version of the original article can be found at https://doi.org/10.1007/s11433-021-1730-x

\footnotetext{
*Corresponding authors (An Pan, email: panan@opt.cn; Caiwen Ma,
} email: cwma@opt.ac.cn) 